\title{
China y sus políticas con América Latina durante el siglo XX
}

China and its policies with Latin America during the 20th century

China e suas políticas com a América Latina durante o século 20

Andrés David Naranjo Navas

Facultad Latinoamericana de Ciencias Sociales - Ecuador adnaranjofl@flacso.edu.ec

https://orcid.org/0000-0002-0835-7544

Bryan Josué Naranjo Navas

Universidad San Francisco de Quito - Ecuador eldestinobryn@hotmail.com

https://orcid.org/0000-0001-5848-5563

Christian Paúl Naranjo Navas

Universidad Nacional de Chimborazo cnaranjo@unach.edu.ec https://orcid.org/0000-0003-1532-203X

\section{RESUMEN}

Este ensayo analiza los intereses políticos y económicos primarios de China en América Latina, tomando en cuenta como punto focal las inversiones chinas en los sectores estratégicos en Venezuela, Brasil, Argentina y Ecuador. El ensayo llega a tres conclusiones: primera, el crecimiento de la influencia de China en la región fue posible por el retiro político de los Estados Unidos; segunda, la creciente necesidad de China de suministrar a sus industrias de materias primas; y, tercera, la generación de deudores que estarían vinculados a China por muchos años.

Palabras clave: China, América Latina, economía, política

\section{RESUMO}

Este ensaio analisa os principais interesses políticos e econômicos da China na América Latina, tendo como ponto focal os investimentos chineses em setores estratégicos na Venezuela, Brasil, Argentina e Equador. O ensaio chega a três conclusões: primeiro, o crescimento da influência da China na região foi possibilitado pela retirada política dos Estados Unidos; segundo, a necessidade crescente da China de fornecer matérias-primas às suas indústrias; e, terceiro, a geração de devedores que ficariam vinculados à China por muitos anos.

Palavras-chave: China, América Latina, economia, política

\begin{abstract}
This essay analyzes the primary political and economic interests of China in Latin America, taking into account the Chinese investments in strategic sectors in Venezuela, Brazil, Argentina and Ecuador as a focal point. The essay reaches three conclusions: first, the growth of China's influence in the region was made possible by the political withdrawal of the United States; second, China's growing need to supply its raw material industries; and third, the generation of debtors that would be linked to China for many years.
\end{abstract}

Key words: China, Latin America, economy, politics

\section{INTRODUCCIÓN}

Las últimas décadas del siglo XX no trajeron a América Latina la prosperidad anunciada por el discurso democrático de Occidente. Las múltiples crisis políticas y económicas abrieron las puertas para considerar otro tipo de gobernanza a través de gobiernos que abrazaron una vieja ideología con una nueva discursiva, el Socialismo del siglo XXI. Estos movimientos se hicieron con el poder político en varios países de América Latina a través de elecciones democráticas. Sin 
embargo, una vez en el poder, estos nuevos gobiernos organizaron asambleas constituyentes que les permitieran estructura un nuevo sistema político. Estos nuevos sistemas macro legales proporcionaron a los presidentes un amplio poder que influyó en el poder judicial y parlamentario, mientras que, al mismo tiempo, construyeron organizaciones y métodos de control sólidos sobre la prensa y las empresas privadas. Los nuevos movimientos socialistas hicieron uso del aparataje de elecciones para justificar los cambios que iban acaeciendo en el resto de la estructura política y económica.

Al mismo tiempo, China se había convertido en una de las superpotencias del mundo después de que experimentara una experiencia devastadora durante la Guerra Fría, cuando la pobreza y el hambre fueron la imagen más relevante de un modelo económico que priorizaba el control y centralización. Desde el cambio de dirección del modelo económico en 1979, China estructuró una economía orientada al libre mercado, con un crecimiento anual promedio de $9.5 \%$ en las últimas cuatro décadas, transformándose en una de las naciones de mayor influencia económica mundial (MORRISON, 2018). Esta tasa de crecimiento permitió duplicar el Producto Interno Bruto cada década, sacando a 800 millones de personas de la pobreza. A principios del siglo XXI, China registraba 3,6\% del PIB mundial, en 2016 su representación era del 15\% del total (VILLAFAÑE, 2016): en 2016, China era el principal productor industrial y agrícola del mundo.

En las últimas décadas, el impacto de China en el desarrollo de la economía mundial no se ha detenido, sino que, por lo contrario, se ha reforzado sobre todo después de la crisis financiera del 2008. Después de 2008, China entró con una fuerza especial en múltiples negociaciones con una gran cantidad de países en desarrollo, especialmente con los países latinoamericanos, quienes habían presenciado un nuevo boom petrolero a partir de 2009: muchos países de América del Sur contrajeron una creciente deuda externa que permitió pagar el aumento del gasto público, esperando que los precios del petróleo se mantengan altos por mucho tiempo.

El crecimiento de una nueva superpotencia encontró una gran necesidad de materias primas que se encontraban en todo el mundo. China combinó su crecimiento con asociaciones estratégicas que le permitieran cubrir sus necesidades en dotación de insumos. La asociación bilateral, causal primordial en la demanda china de materias primas, que a su vez estimuló los precios internacionales, fue una de las principales herramientas utilizadas para acercarse a la región. América Latina encontró un socio muy poderoso, mientras China estaba abierta a pagar altos precios por la materia prima de la región, los países latinoamericanos podrían disponer de fuentes de financiamiento para inversiones en infraestructura, minería, petróleo y electricidad.

Las conversaciones entre los líderes de América Latina y China incluyeron algunos aspectos políticos y económicos de intereses mutuos, especialmente las negociaciones sobre el manejo de los segmentos estratégicos. Cuando los precios de las materias primas llegaron a su punto más alto en 2014, las negociaciones con China se ralentizaron a medida que el presupuesto gubernamental de los países en desarrollo atravesaba un estancamiento prolongado, el valor del intercambio comercial entre América Latina y China disminuyó por tres consecutivos años. Cuando los precios de las materias primas se recuperan en 2017, las exportaciones latinoamericanas aumentaron hasta un $25 \%$ hasta 2018 (INDEX MUNDI, 2019). La misma fuente muestra que el índice de productos básicos de combustible creció 46 puntos desde febrero de 2016 hasta octubre de 2018, el índice de precios del metal aumentó 30 puntos y el índice de precios de los alimentos creció en 10 puntos, y la materia prima agrícola aumentó 3 puntos. Al mismo tiempo, después de 2017, la escena política de los países latinoamericanos abrió espacio para cuestionar los beneficios y costos de la asociación con China.

Según la Comisión Económica para América Latina y el Caribe, CEPAL, el 10\% de las exportaciones de bienes y servicios de la región en 2017 se destinaron a China, mientras que el 19\% de las importaciones provino del país asiático. Se pronostica que en menos de una década, el país 
asiático desplazaría a la Unión Europea como el segundo mayor consumidor de productos de América Latina, por atrás de Estados Unidos, que en 2010 ya había obtenido el primer lugar como el principal exportador a la región.

Este fenómeno chino ocurrió al mismo tiempo que Estados Unidos, antes de completar el segundo año de la administración de Trump en la Casa Blanca, decidiera comenzar una batalla comercial imponiendo aranceles de importación a los productos chinos. China se convirtió en un importante socio político y económico de la región, no solo tenía intereses económicos en América Latina, sino también intereses políticos: el tigre asiático encontró el contexto político perfecto, con el socialismo de los movimientos del siglo XXI, para extender su poder político, cultural y social, llenando el espacio que Estados Unidos había dejado con los gobiernos de la administración Obama.

Dado el relativo aumento de la inversión y la influencia de China y la disminución de la influencia de los Estados Unidos, los países latinoamericanos han encontrado la necesidad de revisar las consecuencias de esta relación comercial y política para tomar mejores decisiones. En este sentido, este artículo analiza los intereses de China en América Latina centrándose en la inversión en los sectores estratégicos de Venezuela, Brasil, Argentina y Ecuador. yos países de América Latina.

\section{FUNDAMENTACIÓN TEÓRICA}

Desde principios del siglo XXI, la influencia de China creció en el desarrollo de la economía mundial y se ha acentuado en las últimas décadas, especialmente con la llegada de algunos líderes socialistas de América Latina, como Hugo Chávez a Venezuela, Lula da Silva en Brasil, Néstor Kirchner y Cristina Fernández de Kirchner en Argentina, y Rafael Correa en Ecuador. Estos nuevos movimientos políticos crearon la imagen del enemigo encarnado en los Estados Unidos, y en cualquier vínculo político que pudiera ser rastreado hasta el hegemón. Al mismo tiempo, el gobierno de Barack Obama inició una política de no intervención: la no intervención construyó el contexto político perfecto a través del cual China entró como el nuevo aliado de la región.

La política de no intervención continuó retóricamente dentro de la campaña de Donald Trump; no obstante, una vez en el poder, Estados Unidos intentó recuperar el espacio político y económico perdido con el apoyo de candidatos políticos y nuevos presidentes que eventualmente reemplazaron las estructuras gubernamentales socialistas, líderes como Mauricio Macri en Argentina, Jair Bolsonaro en Brasil y Sebastián Piñera en Chile. Además, se trabajó en una interminable campaña diplomática y económica para sacar a Nicolás Maduro del autocrático régimen venezolano. El Gobierno de los Estados Unidos enfrentó una retórica contradictoria de proteger las fronteras nacionales y retirarse de los acuerdos comerciales internacionales y al mismo tiempo tratar de recuperar el espacio político perdido durante la administración Obama.

China ha sido un socio comercial importante durante más de dos décadas, con una creciente influencia política, cultural y social que intentó ocupar el espacio creado por el retiro voluntario de la región durante los gobiernos de Obama y luego por la contradictoria retórica y diplomática durante el gobierno de Trump. "Siempre pondré a Estados Unidos en primer lugar, no podemos continuar participando en acuerdos en los que Estados Unidos no obtiene nada bueno" (NBC NEWS, 2017), en menos de un año, el presidente Trump había confirmado su lealtad a su agenda anti globalizante de "Primera América". Trump retiró a los Estados Unidos del Tratado de Comercio con el Pacífico (TPP) con países de Asia y América Latina y el Acuerdo de París, al que están suscritas todas las naciones del mundo. Además, amenazó con poner fin al Acuerdo de Libre Comercio (NAFTA) con México y Canadá, obligando a estos países a firmar un nuevo acuerdo, el Acuerdo Estados Unidos-México-Canadá (USMCA) en el que se enfatiza la protección de la 
propiedad intelectual, el comercio digital, cooperación para programas de anticorrupción, y el comercio de alimentos y agricultura (OFICINA DEL REPRESENTANTE DE COMERCIO DE LOS ESTADOS UNIDOS, 2019). Esta victoria política puso en manifiesto la contradicción entre el discurso de "América primero" y el intento de recuperar una nueva influencia en el ámbito internacional.

Durante los gobiernos de Barack Obama, el socialismo del siglo XXI encontró algunos socios estratégicos en Rusia y, especialmente, en China. La inversión china se viabilizó en sectores estratégicos como el petróleo, la minería y la electricidad a través de ingentes cantidades adeudadas, lo que creó el riesgo de caer en la diplomacia de la trampa de la deuda: los altos intereses proveyeron a China de una influencia importante sobre los países prestatarios (DOLLAR, 2019). El objetivo era prestar dinero a los países en desarrollo para invertir en infraestructura, transporte, energía, suministro de agua y en otros sectores. En 2017, el presidente Xi Jinping señaló que China necesitaba aprovechar las oportunidades que presentaba las tecnologías para desarrollar la interconexión energética global, el desarrollo ecológico bajo en carbono, el avance de la red logística regional y la promoción de políticas, normas y estándares que proporcionen salvaguardas institucionales (XINHUA, 2017).

Según Gallagher y Myers (2020), del Diálogo Interamericano, América Latina tiene 84 préstamos de China, con un total de USD 137 mil millones. Existen 34 préstamos para energía (91,9 mil millones), 34 para infraestructura (26,8 mil millones), 3 para minería (USD 2,1 mil millones). Existen otros 23 tipos de préstamos para comercio, construcción, viviendas y financiamiento fiscal en Venezuela; desarrollo de satélites en Bolivia; y desarrollo de negocios en Uruguay. En los casos de Brasil, Ecuador y Venezuela, China firmó préstamos que tienen la opción de ser pagados en petróleo.

Esta cantidad masiva de préstamos crea la amenaza de caer en la trampa de la deuda que puede desplegarse a través de la administración de sectores estratégicos como la energía o el reembolso en petróleo o material minero. El alto riesgo de caer en la timo de la deuda es más real considerando que los precios del petróleo colapsaron en 2020, registrando precios negativos en abril. La caída de los precios del petróleo ha sido constante desde 2013, de 90 USD por barril $(2010=100)$ a 54 USD en 2019 (BANCO MUNDIAL, 2020). Para los productores petroleros como Brasil, Venezuela y Ecuador, los pocos ingresos de los ingresos petroleros podrían garantizar a China la administración de los sectores estratégicos.

A pesar de la gigantesca cantidad de recursos provenientes de China, no se invirtieron capitales para hacer sostenible la economía, creando una burbuja política basada en la retórica de los gobiernos socialistas latinoamericanos. Vilar (2018) cree que los recursos económicos provenientes de China y, en general, del comercio de materias primas, se desperdiciaron en la propaganda de los gobiernos, en lugar de en la creación de una importante clase media emergente que garantizaría el consumo interno y el desarrollo.

Según Vidal Molina (2019), después de la crisis económica de 2008, un desarrollo crítico comenzó a expandirse, tanto desde el movimiento académico-intelectual como desde los movimientos político-institucionales y sociales, para encontrar una solución a los nuevos problemas que el mundo y, especialmente, la región latinoamericana se enfrentaba. Antes de esta crisis, desde finales de la década de 1990, los movimientos sociales crearon una imagen crítica generada a partir de la implementación de políticas neoliberales. Estos signos dieron espacio para la reconstrucción de una nueva hegemonía política que se construyó en torno a la idea de un nuevo socialismo. Los líderes electos de estos nuevos movimientos socialistas encontraron socios estratégicos en naciones con antecedentes históricos socialistas como China y Rusia.

La crisis financiera del mundo abrió los ojos de la región a una nueva asociación importante con otras naciones poderosas. Mientras que, en el año 2000, China representaba alrededor del $3.6 \%$ 
del PIB mundial, en 2016 su producción trepó al 15\%. China ya era el mayor productor industrial y agrícola del mundo. Los acuerdos bilaterales firmados fueron el principal factor de crecimiento para la región. Según López Villafañe (2016), el modelo chino se ha basado fundamentalmente en mano de obra barata, lo que representó dos dificultades económicas para este país. Para la mayoría de los obreros chinos, su trabajo seguía siendo barato, es decir, sus salarios no aumentaron en la misma tasa que los precios. Esto lleva al problema de la sostenibilidad económica futura de China. El otro problema surge en el objetivo industrial y tecnológico; las empresas chinas siguen siendo competitivas en las antiguas sucursales poco calificadas, caracterizadas por la baja innovación y el desarrollo tecnológico. Esta situación podría cambiar con el plan Made in China 2025 que intenta desarrollar rápidamente inteligencia artificial, comunicaciones, navegación aérea, robótica, biotecnología, electrónica, entre otros.

Ray y Gallagher (2015), en su boletín anual China-América Latina, analizaron los proyectos de nueva generación, el más grande fue el Canal de Nicaragua, más grande que todos los otros proyectos de IED durante los últimos 5 años combinados. A pesar de que el canal planeado nunca comenzó, Ray y Gallagher también mencionan que China registró el 17\% de los nuevos proyectos verdes en América Latina, más que cualquier otro año. En el siglo XXI, la inversión china se centró en proyectos de extracción con el sector de petróleo y gas que representa el $69 \%$ de la inversión china entre 2009 y 2013.

Después de esta gloriosa relación comercial, durante la reducción de los precios del petróleo en 2013, la economía de los países en desarrollo detuvo su crecimiento y el valor del intercambio comercial entre América Latina y China disminuyó durante tres años consecutivos. Sin embargo, en 2017 el valor de las exportaciones latinoamericanas aumentó fuertemente al 25\%, acercándose al nivel más alto alcanzado en 2013 (RAY Y GALLAGHER, 2020). Las relaciones comerciales entre América Latina y China no serían las mismas ya que el entorno político de la región había cambiado: muchos de los líderes socialistas de la región cambiaron democráticamente. Aunque la influencia china disminuyó desde 2017, sus inversiones en las últimas dos décadas fueron tan profundas que, después de la crisis COV19, es muy probable que la asociación continuará con nuevos posibles resultados: algunos países podrían decidir vender o ceder la administración de sus emblemáticas inversiones.

Con la llegada de nuevos gobiernos latinoamericanos, que hicieron claras diferencias con los movimientos socialistas de la primera década del siglo XX, los acuerdos con China se convirtieron en un aspecto crítico de la política local porque muchos se pactaron dentro de una cápsula de hermetismo. Hubo pocos esfuerzos valientes por parte de algunos periodistas para publicar algunos de los puntos importante de los acuerdos comerciales. Fernando Villavicencio (2013), periodista ecuatoriano, publicó un extenso libro sobre las repercusiones políticas y económicas de los acuerdos comerciales entre Ecuador y China. Villavicencio afirmó que el corazón del modelo ecuatoriano era el regreso a un mecanismo de desarrollo extractivo expandido, que concierta el lucro petrolero, la explotación minera, el peaje global, la agricultura de exportación, los biocombustibles, los servicios ambientales y el crecimiento excesivo de la especulación en varios sectores de la economía.

Según Villavicencio, varios de los acuerdos comerciales con China estaban vinculados al pago de créditos petroleros (prepagos), alrededor de 3 mil millones USD, con intereses que oscilan entre $6 \%$ y $7.25 \%$ anual. Además, estos créditos servían como garantía, cobertura y pago inicial para otros créditos. En total, los compromisos con "Petrochina" sumaron más de 421 millones de barriles de petróleo en contratos a largo plazo.

A pesar del cambio del escenario político local, la influencia de China en la región continúa. Según los datos de la CEPAL, el 10\% de las exportaciones de la región van a China, mientras que, en 2017 , el $19 \%$ de las importaciones provenían del país asiático. De esta manera, pronto China 
desplazará a la Unión Europea como el segundo mayor consumidor de productos latinoamericanos. En valores absolutos, las relaciones comerciales muestran que la Unión Europea ya fue desplazada por China. Esto no sucedió con Estados Unidos que sigue siendo el primer socio comercial regional.

\subsection{China y la Política Regional}

China ha demostrado que tiene una estrategia definida, por otro lado, América Latina nunca mostró una estrategia política clara más que la simple retórica política en contra el imperialismo. Desde el comienzo de los gobiernos socialistas latinoamericanos, la asociación con China surgió como un vínculo natural que se desarrolló en el comercio, la asistencia y la cooperación en los esfuerzos energéticos, el apoyo diplomático al discurso político latinoamericano, y la búsqueda de recursos naturales como pagos de inversiones. En resumen, América Latina proporcionó una gran porción de materias primas y dinero para el crecimiento de China. Lafargue y Lafargue (2017) comentan que China no tenía intereses en América Latina durante la mayor parte del siglo XX debido a la abrumadora presencia de Estados Unidos en la región. Sin embargo, desde principios del siglo XXI, esta pasividad cambió, con una presencia creciente en Brasil, Argentina y Chile. Las inversiones en China han alejado la influencia decreciente de Estados Unidos. Aunque la influencia de China sobre la región es innegable, ha demostrado ser un socio poco confiable. De hecho, China no dudó en votar en contra de Brasil cuando este intentó asegurar su participación en el Consejo de Seguridad de la ONU en 2018.

Domínguez Martín (2017) ha argumentado ampliamente que la presencia de China en la política de América Latina es una piedra angular de la preocupación de los Estados Unidos porque la imagen de los Estados Unidos ha cambiado en la región, de un socio poderoso a un autoritario, hegemónico, todopoderoso mal. Si bien la imagen de Estados Unidos es negativa, la imagen de China se ha mantenido neutral, con pocos puntos de discursos políticos contra el poder asiático, pero, en general, con una imagen oculta. Estas variantes han reestructurado el juego político teniendo a los Estados Unidos como el socio malvado hegemónico, todopoderoso, autoritario, por un lado, y China, muy distante para ser importante, como el socio neutral del otro lado. Este aspecto de las relaciones internacionales de la región es especialmente evidente con México, Argentina, Chile, Ecuador y Brasil. Aunque el problema de una disminución de las relaciones políticas entre Estados Unidos y América Latina es real, Domínguez Martín cree que es dudoso que la influencia de los Estados Unidos se degrade más en los próximos años.

Johnson (2017) ha realizado estudios similares, argumentando que la subestimación de la influencia china por parte del gobierno de EE. UU. ha abierto la puerta a la preocupación. La administración del presidente Trump lanzó la Estrategia de Seguridad Nacional en 2017 en la que afirma que China expandió su poder a expensas de la soberanía de otros países, expandiendo los programas de un modelo económico orientado por el estado. La estrategia de seguridad nacional surge como una respuesta al Documento de Política de China sobre América Latina y el Caribe (Xinhua, 2016) que estableció el objetivo de establecer una asociación integral y cooperativa que ofrezca igualdad, beneficio mutuo y desarrollo común con los países de América Latina y el Caribe, mientras se apoya los foros políticos ideológicos como la Comunidad de Estados de América Latina y el Caribe, CELAC.

Algunos académicos utilizaron el término Consenso de Beijing para referirse a dos características fundamentales dentro de la relación económico-política para el desarrollo. La primera característica se basó en un compromiso constante con la innovación y la experimentación. La segunda fue la no fijación del crecimiento del PIB per cápita como objetivo final del modelo de desarrollo, pero la promoción de un modelo de desarrollo sostenible e igualitario. Con esta imagen, China ha sido vista en la región como una mezcla de amenaza y bendición. Por un lado, ha 
aumentado las importaciones de materias primas causando balances comerciales bilaterales con superávit en economías como Chile y Perú, un superávit que se ha acentuado por el aumento en el nivel de precios de los minerales durante el siglo XX. Por ejemplo, el precio de la tonelada métrica de cobre aumentó rápidamente desde la crisis de 1998, de 1,572 USD a 8,828 USD en 2011 (BANCO MUNDIAL, 2020).

La caída del precio del cobre no fue similar al petróleo, el precio promedio del cobre se mantuvo estable alrededor de 6,000 USD hasta 2019. Desde que los gobiernos socialistas fueron cambiados por líderes de una tendencia conservadora, los diplomáticos chinos enviaban misivas en apoyo de los gobiernos socialistas, especialmente a Venezuela. En 2015, CELAC, una organización compuesta por 33 países americanos, expresó su rechazo a las primeras sanciones en contra ce Venezuela, uniéndose al Grupo de 77+ China, que emitió una declaración similar. En 2017, China se unió a quienes escarmientan el discurso belicoso de Estados Unidos contra Venezuela, recordando el principio de no injerencia en otros países, "todos los países deben conducir sus relaciones bilaterales sobre la base de la igualdad, el respeto mutuo y la no injerencia en asuntos internos" (HISPANTV, 2017).

En 2019, China apoyó los esfuerzos realizados por el gobierno venezolano para mantener la soberanía, la independencia y la estabilidad nacional (TAMARA, 2019). En 2020, la vocera del Ministerio de Relaciones Exteriores de China, Hua Chunying, insistió en el sustento de China a la emancipación y soberanía de la República Bolivariana de Venezuela contra la intención de cambiar el gobierno de Maduro por un presidente democrático. Hua Chunying declaró que "Venezuela es un Estado independiente y soberano, China se ha opuesto constantemente a la violación de la soberanía de Venezuela y cualquier interferencia externa en los asuntos internos del país, también nos oponemos a las sanciones unilaterales ilegales" (TELESUR, 2020).

Aunque América Latina no es una prioridad para China, ya que se encuentra en menor importancia que Estados Unidos, Asia o Europa (CORNEJO Y NAVARRO, 2010), China ha tenido una mayor actividad política en la región en la última década. Aunque la importancia de la región en pequeña, América Latina puede hacer mayor peso diplomático en la disputa diplomática con Taiwán. La propuesta china de unificación fue rechazada, incluso el plan conocido como "un país, dos sistemas", en el que Taiwán tendría una independencia significativa si accedía la reunificación china (BBC NEWS, 2019). En 2018, China presionó a varias compañías internacionales para que enlisten a Taiwán como parte de China, amenazando con bloquear cualquier relación comercial. Existe un desacuerdo extendido en la comunidad internacional sobre cómo considerar a Taiwán, ya que tiene su propia constitución con líderes elegidos democráticamente y alrededor de 300,000 tropas activas. Blanchard (2019) ha argumentado que la creciente dependencia económica de las economías de América Latina ha ido de la mano con la extensa influencia política del tigre asiático en la región, China no solo busca su desarrollo económico sino también su influencia política en una región: dejar de lado la influencia de Estados Unidos; la presión política para no reconocer a Taiwán o al Tíbet como regiones independientes; y, la garantía del flujo y la administración de materias primas de América Latina.

\subsection{China y las Economías Latinoamericanas}

En su estudio, Gallagher K. (2016) afirma que el elemento principal entre ambos socios es el comercio, de hecho, China es el principal destino de exportación para países como Brasil, Chile y Perú, y se ha convertido en el segundo país de destino de exportación para Argentina, Costa Rica y Cuba. Este hecho viene de la mano de varios acuerdos bilaterales. Así, China ha asegurado el suministro de materias primas para la próxima década. Este tipo de estrategia surgió de la mano de la deuda, los países de América Latina se endeudaron en cantidades cada vez mayores, muchas de 
las cuales se pagarán a través de las exportaciones de materias primas, especialmente a través del comercio de petróleo.

Por su vez, Gil Barragán y Aguilera Castillo (2017) creen que China ha jugado un doble papel en la región: por un lado, China es el socio comercial más importante a través del incremento de la inversión extranjera directa; por otro lado, China se ha convertido en un competidor de la manufactura. Este tipo de relaciones comerciales ha estructurado los resultados en cuatro maneras posibles: primero, la demanda china de materias primas ha creado una posición débil para América Latina debido a las fluctuaciones de precios; segundo, las inversiones chinas han empujado a los países a una mutación de la producción; tercero, el sector manufacturero de la región está en riesgo debido a los competitivos sectores chinos que se han implementado en las industrias petroleras y mineras; cuarto, China tiene una creciente participación de mercado en el sector manufacturero, lo que afecta las exportaciones de los países latinoamericanos.

Johnson (2017) menciona que una buena parte del nivel de vida de la región depende de la entrada de recursos económicos que provienen de China. Del mismo modo, Hiratuka (2018) afirma que los flujos económicos chinos son notables en la inversión extranjera directa, en los flujos de financiación y en los proyectos de infraestructura. Estos factores son más importantes que el comercio, "los cambios posteriores a la crisis han provocado que la tasa de crecimiento de las exportaciones disminuya debido a la caída de los precios de los productos básicos y la desaceleración del crecimiento de la economía china. Sin embargo, la acumulación de capacidad ociosa y el estancamiento de la demanda mundial significan que las importaciones de China continuaron aumentando y solo se vieron interrumpidas por la fuerte desaceleración reciente en las economías de América Latina" (HIRATUKA, 2018, p. 20).

La región enfrenta dos desafíos claros. Primero, el desafío creado por la dependencia creciente de China en tres áreas: comercio, infraestructura e inversión extranjera directa. Esta dependencia se hizo evidente en la crisis económica de 2020. Segundo, los movimientos socialistas son muy activos: las huelgas generales en América Latina a fines de 2019 mostraron el poder político y logístico de algunos grupos socialistas. Estos desafíos proponen un nuevo escenario del futuro de la región y sus próximas pruebas políticas y económicas. Como se ve en la figura 1, Perroti (2015) afirma que la demanda china de productos primarios latinoamericanos se ha originario de su proceso de industrialización, en el que los metales han jugado un rol predominante. La evolución de este proceso no solo ha llevado a aumentos en las cantidades demandadas, sino que también ha causado una importante presión al alza sobre los precios de los bienes primarios y minerales, lo que se ha traducido en una mejora importante en los términos de intercambio. Esto ha sido el resultado del vertiginoso proceso de industrialización del país. La demanda de metales también ha sido promovida por la construcción y otros proyectos de infraestructura.

Figura 1. El impacto de la demanda china en los precios mundiales en porcentajes, 2007 


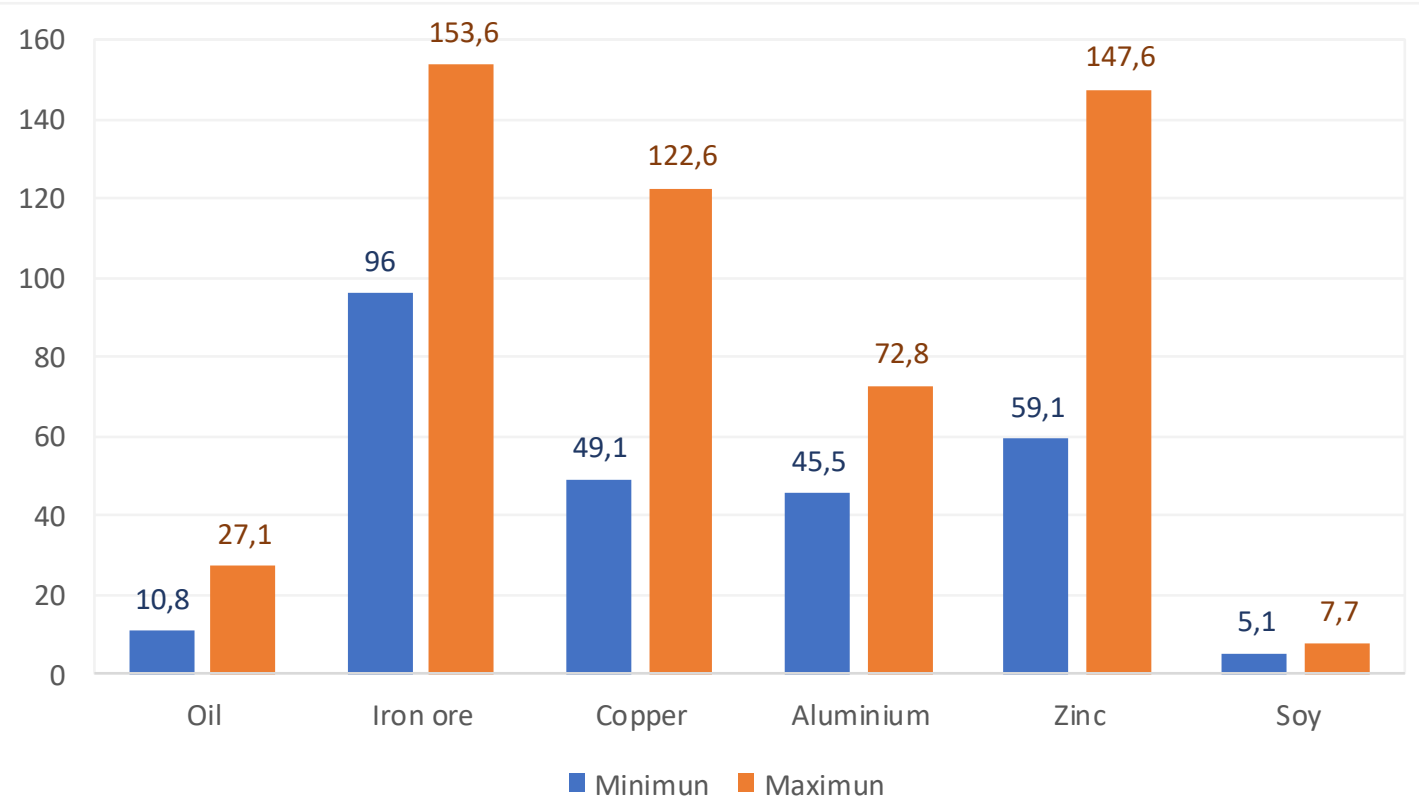

Fuente: Perroti, 2015, p. 50.

China lidera el consumo del sector primario como insumos para la producción de manufactura y las mejoras tecnológicas. También ha habido avances importantes en el sector de servicios en el que el país asiático ha aumentado su eficiencia, especialmente en el comercio (transporte, infraestructura física, comunicaciones, servicios comerciales y profesionales, incluidos los servicios financieros). El papel de esta economía como consumidor global también es sustantivo: China compra el 53\% de las ventas externas de soja, el 28\% de aceite de soja y el $23 \%$ de algodón, y es el primer lugar como consumidor global de carbón, estaño y zinc y cobre (PERROTI, 2015). En relación con esto último, su participación en el consumo mundial de minerales y metales fue de alrededor del $40 \%$ en 2009 en plomo, níquel, estaño, zinc, acero primario, cobre refinado y aluminio. El mismo año, consumió el $10 \%$ del petróleo en todo el mundo.

\section{RESULTADOS Y DISCUSIÓN}

Según Ortiz (2017), desde 2010 el flujo promedio de inversión extranjera directa a la región ha sido cercano a USD 172,000 millones, el doble del flujo promedio recibido entre 2001-2009, pero con una caída acumulada de 26.6\% entre 2011 y 2016. En este contexto, la inversión extranjera directa china estimada pasó de una cantidad anual promedio de USD 1,357 millones entre 2001-2009 a USD 10,817 millones en 2010-2016, la inversión extranjera directa china alcanzó su tercer nivel más alto en 2016. Durante 2001-2016, las compañías chinas realizaron 274 actividades comerciales con los países de la región, con un flujo acumulado de USD 87,928 millones. El empleo estimado asociado con las inversiones fue de $202 \mathrm{mil}$ empleos (ORTIZ, 2017, p. 10).

El período 2010-2016 fue testigo del máximo dinamismo, acumulando USD 76,000 millones y 168,806 empleos. Sin embargo, durante 2001-2016, alrededor de 7 de cada 10 transacciones estuvieron vinculadas a procesos de aumento de capital, las nuevas inversiones representaron solo el $36.46 \%$ de la IED y el $49.85 \%$ del empleo. En contraste, las fusiones y adquisiciones presentaron un monto por transacción cinco veces mayor que el reportado por las nuevas inversiones $\mathrm{y}$ representaron más de dos tercios del PIB chino total. 
Se analizarán las inversiones de China en cuatro países diferentes: Venezuela, Brasil, Argentina y Ecuador. Estos países han recibido la mayor parte de las inversiones de China en la región. El monto total de las inversiones en estos países suma un total de USD 126.6 mil millones de 2007 a, China se ha convertido en el principal socio de inversión y se ha convertido en el principal prestamista, con un total de 55 préstamos (GALLAGHER y MYERS, 2020). Según la misma base de datos, en los últimos años solo se registraron cuatro nuevos préstamos debido a los pocos ingresos que estos países tuvieron en la crisis de precios de las materias primas.

\subsection{Venezuela}

El gobierno de Hugo Chávez trató de evitar cualquier contrato de inversión con los Estados Unidos, a quién consideraba como la reencarnación del diablo, y trató de fomentar relaciones más profundas con otros gobiernos como el gobierno de Rusia, China, etc. Desde 2007, como se ve en la figura 2, China ha aumentado sus inversiones en Venezuela, especialmente en dos sectores: energía e infraestructura, asignando a China como el principal actor regional. Las inversiones chinas en Brasil representan menos de la mitad de las inversiones en Venezuela, las inversiones en Ecuador y Argentina representan menos de un tercio.

Con el aumento de la influencia de China, Venezuela perdió la independencia y la autonomía política, dado la creciente cantidad de deuda que se estaba adquiriendo. De hecho, algunos académicos calculan la deuda externa en USD 246 millones (TRADING ECONOMICS, 2019). Las inversiones de China en Venezuela han abierto la puerta a acuerdos por debajo de la mesa que han llevado la estructura política a altos niveles de corrupción. Según el ex fiscal general venezolano Tarek William Saab, hasta el 2018, Venezuela ha perdido USD 15 mil millones debido a la corrupción (Sputnik Mundo, 2018).

La inversión económica de China en la región se centra en el acceso a las materias primas, lo que hace que América Latina sea dependiente del mercado externo. La gestión particular de China de la política exterior es llevar a cabo una agenda diplomática pragmática, especialmente en países en desarrollo que tienen recursos importantes, especialmente energía. Los acuerdos bilaterales con Venezuela cubren áreas como energía, infraestructura, agricultura, tecnología (ROSALES, PÉREZ, PRADO Y BÁRCENA, 2015). En 2019, después de dos décadas de socialismo, Venezuela se ha convertido en un país de alto riesgo para las inversiones, incluidas las inversiones de China: la única forma en que China seguiría introduciendo dinero en Venezuela se basa en la adquisición de una fuente estable de petróleo y productos mineros.

Figura 2. Inversiones chinas en Venezuela, millones de dólares 
25000

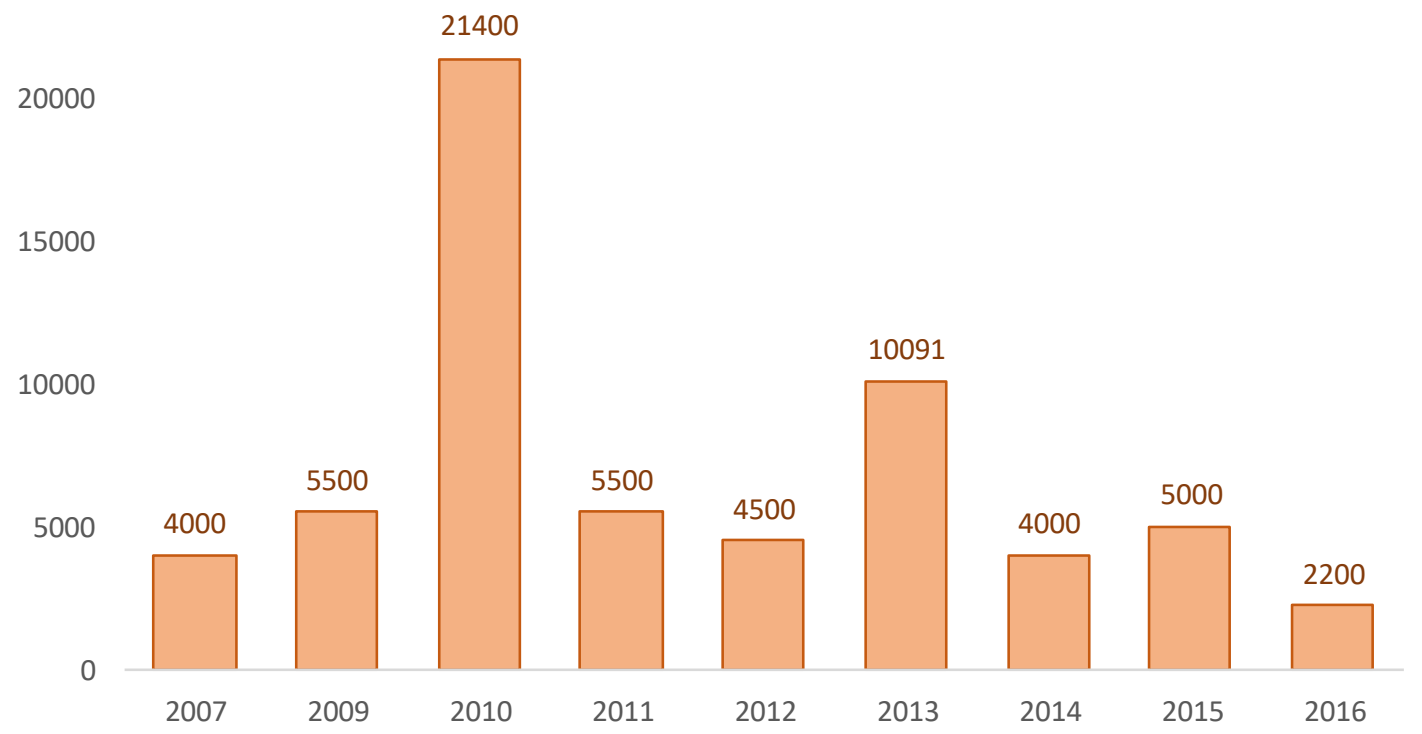

Fuente: apéndice 1.

\subsection{Brasil}

Brasil es probablemente el principal actor político y económico de la región. El aumento de las inversiones de China en Brasil coincide con la tendencia en Venezuela: este elemento es comprensible dado que Venezuela, Brasil, Argentina y Ecuador estaban siendo liderados por gobiernos socialistas. El gobierno de Lula da Silva abrió las puertas a la alta influencia y dependencia de las inversiones de China; no obstante, el aparato industrial del país no estaba bajo persecución, como sucedió en el caso de Venezuela. Aunque el aparato industrial del país tuvo que retener la presión de impuestos y derechos laborales más altos, pudo seguir con los niveles de producción.

Como se ve en la figura 3, Brasil recibió una inversión total de USD 28.9 mil millones de 2007 a 2019. Las inversiones de China en Brasil coinciden con la inestabilidad de la economía brasileña, desde el comienzo del siglo, el crecimiento económico de Brasil ha oscilado entre crecimiento y recesión. En las últimas décadas, el único período en el que Brasil pudo considerar un cierto crecimiento económico fue de 2002 a 2008, el crecimiento promedio anual de Brasil fue de 4.8\%, combinado con una inflación de 6\% entre 2004 y 2008 (REMES LENICOV, VIOLA Y KNOLL, 2015, p. 3). Además, tomando en cuenta la grave crisis que provocó el COV-19, para 2021, Brasil enfrenta la difícil situación de equilibrar sus finanzas mientras baila con los extremos del espectro político.

Figura 3. Inversiones chinas en Brasil, millones de dólares 


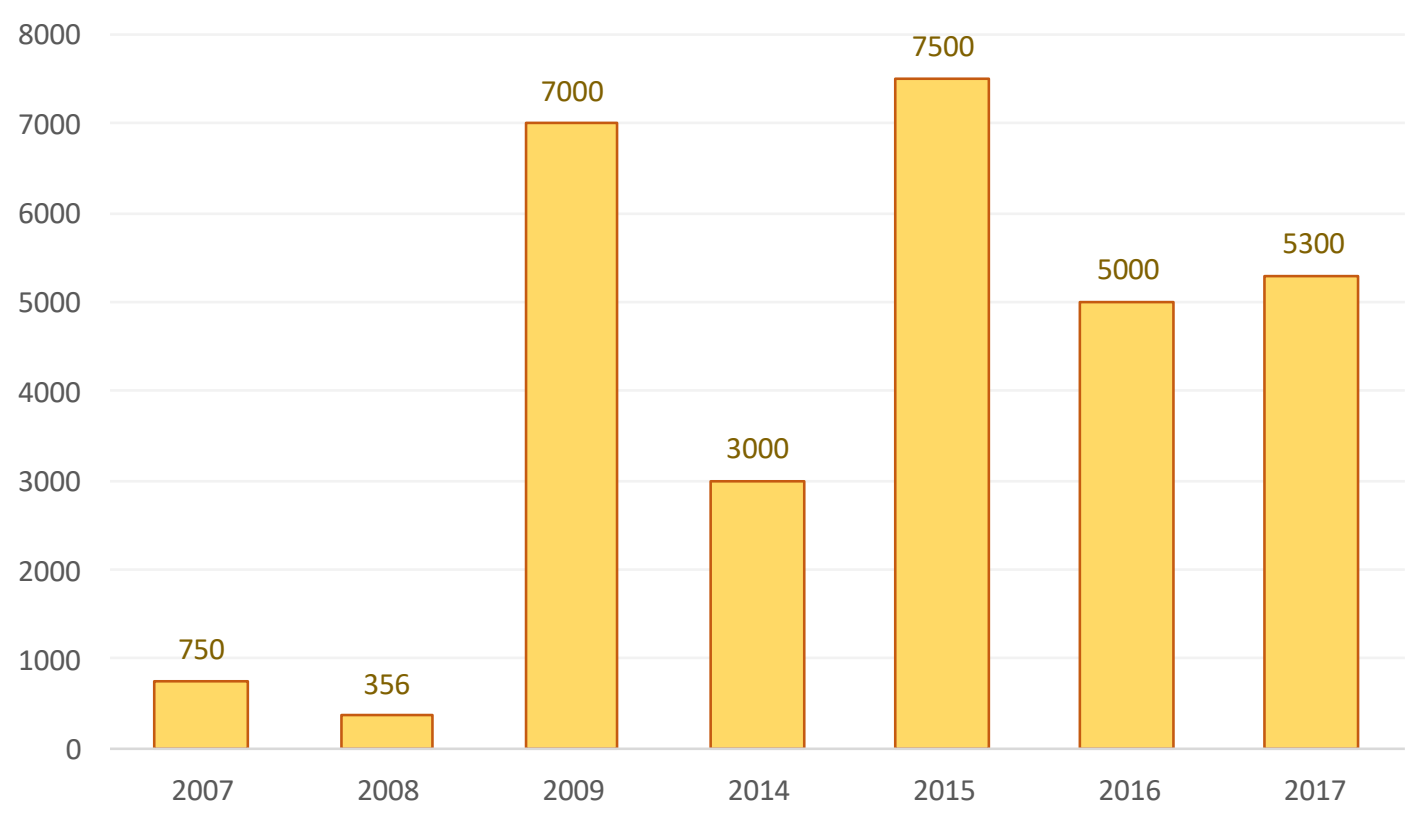

Fuente: apéndice 2.

\subsection{Argentina}

Argentina es considerado como un país que recibió la gran influencia de la migración europea durante el siglo pasado, especialmente en el período entre guerras. Argentina ha tenido una larga tradición de acercamiento y pronto alejamiento de las naciones desarrolladas. En el primer caso, se recuerda la presidencia de Carlos Menem, del último caso, está claro el caso de Juan Domingo Perón. Desde el comienzo del nuevo siglo, Argentina se separó de los países desarrollados durante la presidencia de Néstor y Cristina Kirchner. De 2003 a 2015, las inversiones de China fueron bienvenidas en la nación como una forma de salir de la influencia de los países cercanos al imperio.

Según Remes Lenicov, Viola y Knoll (2015), Brasil es el primer destino de las exportaciones argentinas y China el tercero, después de la Unión Europea (UE). Como se ve en la figura 4, las inversiones de China en Argentina ascienden a un total de 17.1 mil millones de dólares. Durante 2010-2015, la inversión extranjera directa de China se triplicó, con una diversificación progresiva de inversiones hacia la energía, petróleo y gas (15\%), minería (19\%) y agricultura (4\%), que en conjunto representan el 38\% del total (Oviedo, 2017, p. 101). Después del gobierno de Mauricio Macri, con el regreso al gobierno socialista de Alberto Fernández, Argentina se enfrenta a la recuperación de altos niveles de deuda externa y altos niveles de gasto social que no se respaldan en un equilibrio fiscal coherente.

Figura 4. Inversiones chinas en Argentina, millones de dólares 


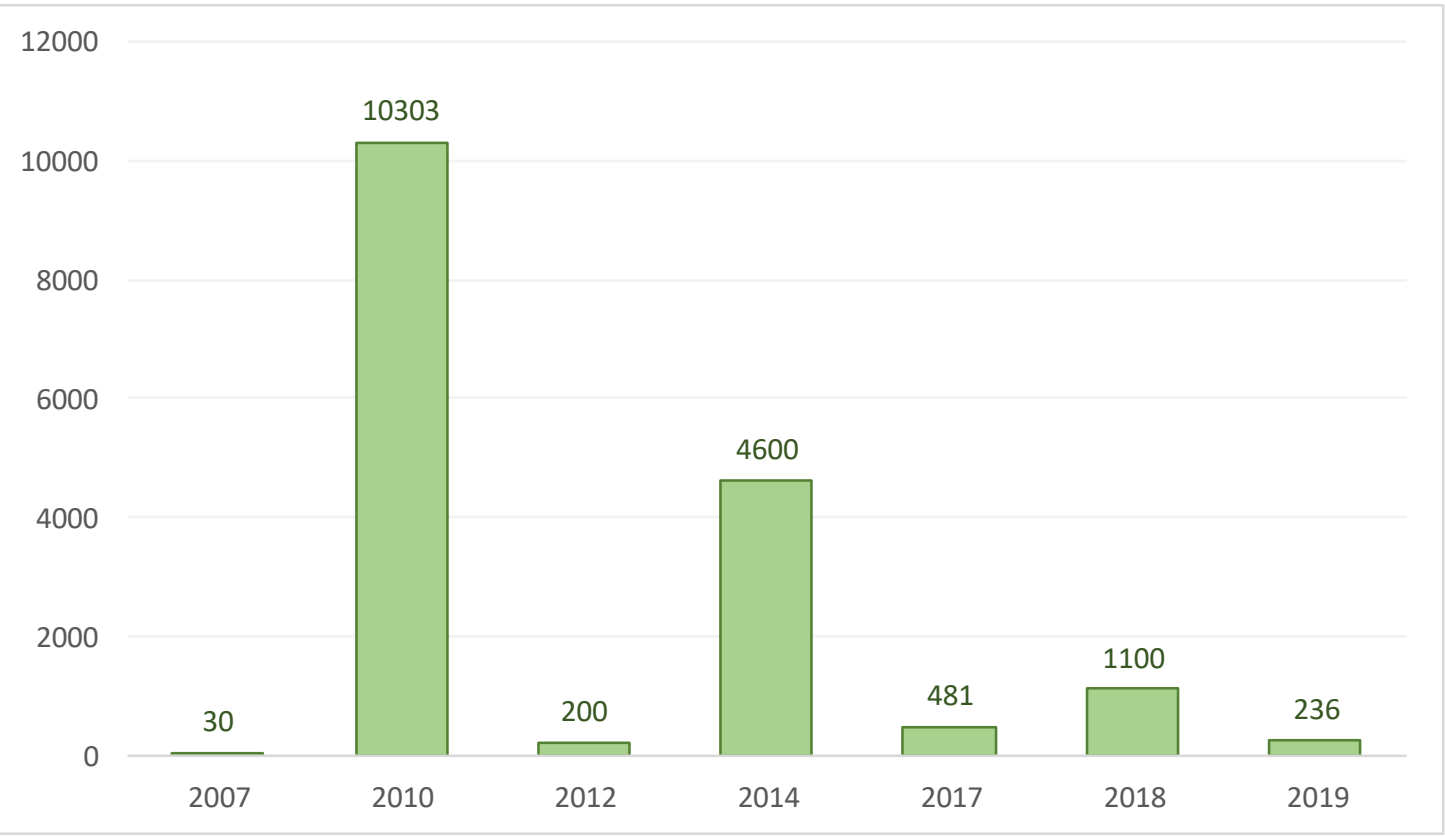

Fuente: apéndice 3

\subsection{Ecuador}

Ecuador vino de una década de una situación política convulsionada antes del ascenso al poder de Rafael Correa. Tres presidentes fueron derrocados en menos de diez años, lo que preparó el contexto para la llegada de un candidato que culparía de todos los males del país a la influencia del hegemón y a la influencia de los empresarios. Rafael Correa llegó al poder con un discurso que alejó al país de las inversiones estadounidenses, de hecho, de grandes inversiones de los países occidentales, así China llegó como el socio comercial perfecto.

La llegada de China al país abrió la puerta a altos niveles de corrupción, que no son exclusivos del país, sino que forman parte del patrón común entre los países latinoamericanos que fueron impulsados por el socialismo del siglo XXI. Las campañas publicitarias muy costosas fomentaron la idea de una nueva nación con nuevas fuentes de energía, y con inmensas inversiones que cambiarían el modelo industrial de la nación. Las inversiones en China sirvieron como plataforma política para embellecer la grandeza del discurso revolucionario.

Según Luzuriaga (2017), en un contexto de difícil financiamiento para América Latina, China se consolidó como un socio estratégico de Ecuador a través de préstamos con condiciones extremadamente laxas. Esta relación era apropiada para la influencia política y económica de China en la región ya que le permitía asegurar los recursos naturales necesarios para posicionar a sus empresas. Como se ve en la figura 5, la inversión de China en Ecuador asciende a un total de 18.4 mil millones de dólares, lo que configura a China como el principal socio acreedor de la nación.

Figura 5. Inversiones chinas en Ecuador, millones de dólares 


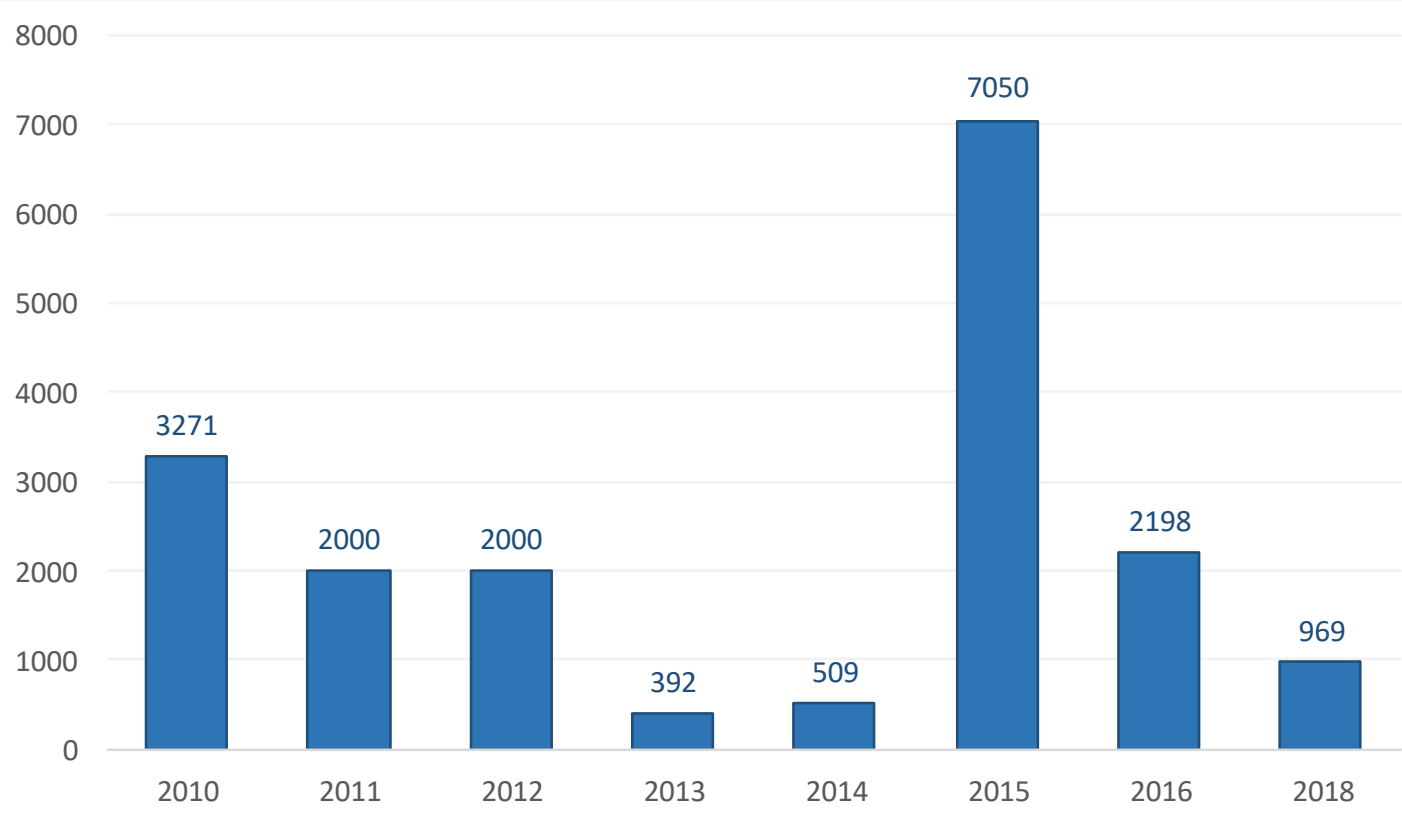

Fuente: apéndice 4

En las últimas dos décadas, China ha pasado de ser un pequeño socio político y económico de la región a ser uno de los principales. Este cambio tiene que ver con dos factores importantes: primero, la retirada de los Estados Unidos como el principal actor político de la región en las últimas dos décadas; segundo, la intención de encontrar fuentes de materias primas. El primer elemento fue visible durante los gobiernos de Barack Obama, que hicieron muy poco para detener el creciente crecimiento del socialismo en la región y para detener la creciente afluencia de las inversiones de China. En referencia al segundo punto, China ha jugado un papel muy inteligente al ofrecer a los países de la región grandes cantidades de dinero con la condición de tener el monopolio de áreas como la minería, el petróleo y la energía. Con este panorama, y considerando la crisis del COVID-19, es probable que China tome el control administrativo de algunas de sus inversiones en América Latina.

La creciente exportación de capital de China a América Latina desde el 2000, que ha tomado la forma de planes de infraestructura, financiamiento e inversión extranjera directa, ha motivado el interés en el sector académico para comprender sus motivaciones, comportamiento y repercusiones socioeconómicas y ambientales. La región también ha ganado peso como socio comercial de China. En 2000, absorbió el 3\% de las exportaciones totales de bienes de China y fue el origen del $2 \%$ de sus importaciones, mientras que en 2013 su participación en ambos flujos ascendió a 6\% y 7\%, respectivamente; además, alrededor del $13 \%$ del petróleo importado a China proviene de Venezuela y Brasil (RÍOS, 2019). A pesar de ello, en comparación con otras regiones, América Latina y el Caribe no se encuentra entre los socios comerciales más importantes para China. Los intercambios bilaterales, estimulados por la demanda de materias primas de China, impulsaron los precios y se convirtieron en el motor de crecimiento para la región.

En 2014, la economía de los países en desarrollo se estancó y el valor de cambio de la región con China cayó durante tres años consecutivos. En 2017, se expandió nuevamente con un crecimiento importante, acercándose al máximo histórico alcanzado en 2013; no obstante, la crisis sanitaria del 2020 allanó el camino hacia una situación económica muy crítica. Los países de América Latina y el Caribe son fuentes de recursos naturales y productos básicos en el intercambio mundial. Si bien toda la región representa alrededor del 6\% del comercio mundial, las exportaciones chinas se concentraron en Asia (50\%), Estados Unidos (20\%), Europa (19\%), y 
América Latina, África y Oceanía (11\%). Con respecto a las importaciones de China, el 57\% corresponde a Asia, el 18\% a Europa, el $10 \%$ a los EE. UU. y el 15\% a América Latina, África y Oceanía. El 68\% de la inversión china se realizó en Asia, el 13\% en Estados Unidos y Europa, el $12 \%$ en América Latina, el $4 \%$ en África y el 3\% en Oceanía.

\section{CONCLUSIONES}

Es escrito propone una revisión histórica de los hechos y relaciones políticas surgidas a partir de la intervención en China como fuente de financiamiento de los proyectos de inversión y gasto en los países de América Latina. Con este propósito, se hace uso del método histórico y comparativo para la contextualización de las relaciones políticas y para la revisión de los intereses alrededor de la inversión China.

El modelo chino se ha basado fundamentalmente en la mano de obra barata, creando dos problemas económicos. El trabajo de los trabajadores chinos sigue siendo barato, es decir, sus salarios no han aumentado lo suficiente, y esto lleva al problema de la sostenibilidad del modelo. Algunos economistas chinos lo llaman la trampa del país de ingresos bajos y moderados, lo que dificultará que China pase a ser un país desarrollado. Esto también limitará el potencial del mercado interno a largo plazo. Incorporar año tras año la enorme reserva laboral con bajos salarios, pondrá a China permanentemente en la condición de ser una economía de bajo costo laboral indefinidamente. Por otro lado, durante mucho tiempo, la posición de China como socio clave para América Latina se ha subestimado, limitándolo en gran medida a un papel creciente e importante en el comercio y la inversión, pero subestimando su papel político.

El camino acelerado de los años anteriores se complementó con la firma de acuerdos de asociación estratégica generalizados con los gobiernos socialistas de Argentina, Brasil, Ecuador y Venezuela. Así, América Latina es parte de los objetivos futuros de China, además representa una oportunidad para que la región mejore su infraestructura, su capacidad productiva, la formación de cuadros técnicos, incluido el desarrollo de una economía más atenta al medio ambiente y la mejora de la conectividad y la gobernanza.

China fue percibida como un actor que ayudó a mejorar la estabilidad en la región hasta la retirada de algunos gobiernos socialistas. Después de la reducción del precio del petróleo y el cambio de gobiernos, la imagen de China ha crecido en persistencia de dudas y reservas de sus intenciones. Como se ve en esta investigación, la influencia de China ha aumentado en las últimas dos décadas debido a tres elementos importantes. Primero, las últimas administraciones ejecutivas de Estados Unidos jugaron un papel importante al decidir alejarse de la política regional. Era comprensible haber previsto el surgimiento de movimientos socialistas guiados por los modelos políticos y económicos de Cuba y Venezuela. Este tipo de regímenes intentó apartarse de cualquier asociación con los Estados Unidos e intentó iniciar nuevas relaciones con otros países, especialmente con China, el país de crecimiento más rápido en las últimas décadas.

Segundo, el interés de China para asegurar el suministro de materias primas. Las inversiones en China se pueden dividir en tres áreas: petróleo, minería y electricidad. Las dos primeras áreas son extremadamente importantes para asegurar una industria y economía en crecimiento. Finalmente, China ha asegurado su influencia en la región durante, al menos, otra década, dado el tipo de contratos de deuda que firmaron los países latinoamericanos. Países como Venezuela, Bolivia, Ecuador, Nicaragua, Argentina y Brasil se endeudaron con China con reembolsos que se acordaron con exportaciones de petróleo. En la mayoría de casos, estos reembolsos se programaron en un período de 5-10 años.

Los límites del estudio se enmarcan en el rápido cambio de la situación política en la región y el cambio de la influencia de los Estados Unidos con el cambio de gobierno. Se puede prever que 
China intente seguir influyendo en la región a través de los gobiernos del socialismo del siglo XXI, es sus antiguas y nuevas generaciones; sin embargo, existen intentos de alejarse de esta influencia como es el caso de Ecuador que ha recibido ayuda económica de la Corporación Financiera de Desarrollo Internacional (EE.UU.) para pagar ciertos tramos de la deuda con China. Las futuras agendas de investigación pueden enfocarse en estos cambios de políticas públicas, además de la crisis sanitaria que ha provocado problemas fiscales en la mayoría de países de América Latina.

\section{REFERENCIAS}

BBC NEWS. What's behind the China-Taiwan divide?, 2 de enero de 2019, Recuperado el 04 de May de 2020, de https://www.bbc.com/news/world-asia-34729538

BLANCHARD, Jean-Marc F. The Politics of Latin America's Investment and Other Links with China: Contextualizing the Region's Cash Chasing while Racking Richer Research Rewards. Journal of Chinese Political Science, 24(4), 565-582, diciembre de 2019.

CORNEJO, Romer, y NAVARRO, Abraham. China y América Latina: recursos, mercados y poder global. Nueva Sociedad (228), 79-99, 2010.

DOLLAR, David. Understanding China's Belt and Road Infrastructure Projects in Africa. Brookings Institution, 2019.

DOMÍNGUEZ MARTÍN, Rafael. La Princesa y el Dragón: Cooperación China en América Latina y más allá. Revista Internacional de Cooperación y Desarrollo, 4(2), 3-27, 2017.

GALLAGHER, Kelvin. The China Triangle. Latin America's China Boom and the Fate of the Washington Consensus. Oxford: Oxford University Press, 2016.

GALLAGHER, Kelvin, y MYERS, Margaret. China-Latin America Finance Database. Recuperado el 1 de may de 2020, de The Dialogue Leadership for the Americas: https://www.thedialogue.org/map list/ , 2020.

GIL BARRAGÁN, Juan Manuel, y AGUILERA CASTILLO, Andrés. China y América Latina: ¿Socios estratégicos o competidores? Negocios Internacionales(82), 73-90, 2017.

HIRATUKA, Celio. Changes in the Chinese Development Strategy after the Global Crisis and its Impacts in Latin America. Revista de Economía Contemporánea, 22(1), 1-25, 2018.

HISPANTV. China advierte a EEUU contra intervención en Venezuela, 14 de agosto de 2017. Recuperado el 04 de May de 2020, de https://www.hispantv.com/noticias/china/350436/venezuela-eeuu-trump-opcion-militarinjerencia

INDEX MUNDI. Commodity Prices. Obtenido de https://www.indexmundi.com/commodities/ , 2019.

JOHNSON, Kendall. The New Middle Kingdom China adn the Early American Romance of Free Trade. Baltimore: Johns Hopkins University Press, 2017.

LAFARGUE, Francois y LAFANGUE, Vincent. Objectif Concours Géographie Économique Cat A et B 2017. Paris: Hachette Educ, 2017.

LÓPEZ VILLAFAÑE, Víctor. La emergencia del China como potencia mundial. Fin del período de alto crecimiento y nuevos desafíos. Coyuntura y debate (26), 168-196, 2016.

LUZURIAGA, Miguel. Inversiones China en Ecuador: Andres Petroleum y los Bloques 79 y 83. Quito: Centro de Derechos Económicos y Sociales, 2017.

MORRISON, Wayne. China's economic rise: history, trends, challenges, and implications for the United States. Congressional Research Service, 2018.

NBC NEWS. 'America First': Read Trump's Full United Nations Speech. Obtenido de https://www.nbcnews.com/politics/white-house/america-first-read-trump-s-full-united-nations-speech-n802676 , 2017.

OFFICE OF THE UNITED STATES TRADE REPRESENTATIVE. Agreement between the United States of America, the United Mexican States and Canada. Obtenido de https://ustr.gov/trade-agreements/free-tradeagreements/united-states-mexico-canada-agreement/agreement-between, 13 de diciembre de 2019.

ORTÍZ, Samuel. Inversión extranjera directa de China en América Latina y el caribe, aspectos metodológicos y tendencias durante 2001-2016. Economía informa, 406, 4-17, octubre de 2017.

OVIEDO, Eduardo Daniel. Inversiones de China, Corea y Japón en Argentina. Rosario: UNR Editora, 2017.

PERROTI, Daniel. La República Popular de China y América Latina: impacto del crecimiento económico chino en las exportaciones latinoamericanas. Revista CEPAL (116), 48-61, 2015.

RAY, Rebecca, y GALLAGHER, Kevin. China-Latin America Economic bulletin 2015 Edition. Global Economic Governance Iniciative, 2015.

REMES LENICOV, Jorge, VIOLA, Anahi, y KNOLL, Patricia. Las Economía de Brasil y China. Observatorio de la Economía Mundial (19), 1-16, 2015.

RÍOS, Xulio. El estado de las relaciones China- América Latina. Agenda 2030, 2019.

ROSALES, Osvaldo, PÉREZ, Ricardo, PRADO, Antonio. y BÁRCENA, Alicia. América Latina y el Caribe y China. Hacia una nueva era de cooperación económica. Santiago de Chile: CEPAL, 2015. 
SPUTNIK MUNDO. La corrupción deja pérdidas de \$15.000 millones a Venezuela, 21 de febrero de 2018. Recuperado el 02 de mayo de 2020, de https://mundo.sputniknews.com/america-latina/201802211076478559-caracascasos-corruptos/

TAMARA, Gil. Crisis en Venezuela: qué intereses tiene China en el país latinoamericano (y por qué tiene tanto que perder), 14 de febrero de 2019. Recuperado el 04 de mayo de 2020, de BBC News: https://www.bbc.com/mundo/noticias-america-latina-47221713

TELESUR. China reitera apoyo a Venezuela ante sanciones estadounidenses, 20 de marzo de 2020. Recuperado el 03 de mayo de 2020, de Telesurtv.net: https://www.telesurtv.net/news/china-reitera-apoyo-venezuela-202003300012.html

THE WHITE HOUSE. National Security Strategy. Washington, 2017.

THE WORLD BANK. Commodity Markets, 23 de enero de 2020. Recuperado el 01 de May de 2020, de Pink Sheet Data Annual prices: https://www.worldbank.org/en/research/commodity-markets

TRADING ECONOMICS. Venezuela Public External Debt, 2019. Recuperado el 01 de May de 2020, de https://tradingeconomics.com/venezuela/external-debt

VIDAL MOLINA, Paula. Neoliberalismo, neodesarrollismo y socialismo bolivarian. Modelos de desarrollo y pol'iticas p'ublicas en Am'erica Latina. Santiago de Chile: Ariadna Ediciones, 2019.

VILAR, José Rafael. Auge y caída del socialismo del siglo XXI. Revista gobernanza (44), noviembre de 2018.

VILLAVICENCIO, Fernando. Ecuador made in China. Quito: Interamerican institute for Democracy Fondo Editorial, 2013.

XINHUA. Full text of President Xi'speech at opening of Belt and Road forum, 14 de mayo de 2017. Recuperado el 3 de mayo de 2020, de XinhuaNet: http://www.xinhuanet.com//english/2017-05/14/c_136282982.htm

XINHUA. Full text of China's Policy Paper on Latin America and the Caribbean, 24 de noviembre de 2016. Recuperado el 02 de mayo de 2020, de http://www.china.org.cn/world/2016-11/24/content_39777989.htm

\section{Apéndices}

Apéndice 1. Inversiones chinas en Venezuela, millones de dólares

\begin{tabular}{|c|c|c|c|}
\hline Año & Clase & Prestamista & Cantidad (millones de dólares) \\
\hline 2007 & Energía & China Developmnet Bank & 4000 \\
\hline 2009 & Energía & China Developmnet Bank & 4000 \\
\hline 2009 & Minería & China Developmnet Bank & 1000 \\
\hline 2009 & Energía & China Ex-Im Bank & 500 \\
\hline 2010 & Otro & CDB and Portugal's BES & 1100 \\
\hline 2010 & Energía & China Developmnet Bank & 20300 \\
\hline 2011 & Energía & China Developmnet Bank & 4000 \\
\hline 2011 & Energía & China Developmnet Bank & 1500 \\
\hline 2012 & Energía & China Developmnet Bank & 500 \\
\hline 2012 & Energía & China Developmnet Bank & 4000 \\
\hline 2013 & Energía & China Developmnet Bank & 4000 \\
\hline 2013 & Minería & China Developmnet Bank & 700 \\
\hline 2013 & Infraestructura & China Ex-Im Bank & 391 \\
\hline 2013 & Energía & China Developmnet Bank & 5000 \\
\hline 2014 & Infraestructura & China Ex-Im Bank & 4000 \\
\hline 2015 & Energía & China Developmnet Bank & 5000 \\
\hline 2016 & Energía & China Developmnet Bank & 2200 \\
\hline Total & & & $\$ 62191$ \\
\hline
\end{tabular}

Fuente: Gallagher y Myers, 2020

Apéndice 2. Inversiones chinas en Brasil, millones de dólares

\begin{tabular}{cccc}
\hline Año & Clase & Prestamista & Cantidad (millones de dólares) \\
\hline 2007 & Energía & China Developmnet Bank & 750 \\
2008 & Energía & China Developmnet Bank & 356
\end{tabular}




\begin{tabular}{cccc}
2009 & Energía & China Developmnet Bank & 7000 \\
2014 & Energía & China Developmnet Bank & 3000 \\
2015 & Energía & China Developmnet Bank & 1500 \\
2015 & Infraestructura & China Developmnet Bank & 1200 \\
2015 & Energía & China Developmnet Bank & 3500 \\
2015 & Otro & China Ex-Im Bank & 1300 \\
2016 & Energía & China Developmnet Bank & 5000 \\
2017 & Otro & China Ex-Im Bank & 300 \\
2017 & Energía & China Developmnet Bank & 5000 \\
\hline Total & &
\end{tabular}

Apéndice 3. Inversiones chinas en Argentina, millones de dólares

\begin{tabular}{|c|c|c|c|}
\hline Año & Clase & Prestamista & Cantidad (millones de dólares) \\
\hline 2007 & Otro & China Developmnet Bank & 30 \\
\hline 2010 & Otro & China Developmnet Bank & 30 \\
\hline 2010 & Infraestructura & CDB and others & 10000 \\
\hline 2010 & Infraestructura & CDB and CITIC & 273 \\
\hline 2012 & Energía & China Developmnet Bank & 200 \\
\hline 2014 & Energía & China Developmnet Bank & 2500 \\
\hline 2014 & Infraestructura & Belgrano Cargas train line & 2100 \\
\hline 2014 & Otro & China Ex-Im Bank & 162 \\
\hline 2017 & Otro & SME development & 150 \\
\hline 2017 & Energía & China Ex-Im Bank & 331 \\
\hline 2018 & Infraestructura & China Ex-Im Bank & 1100 \\
\hline 2019 & Infraestructura & China Developmnet Bank & 236 \\
\hline
\end{tabular}

Fuente: Gallagher y Myers, 2020

Apéndice 4. Inversiones chinas en Ecuador, millones de dólares

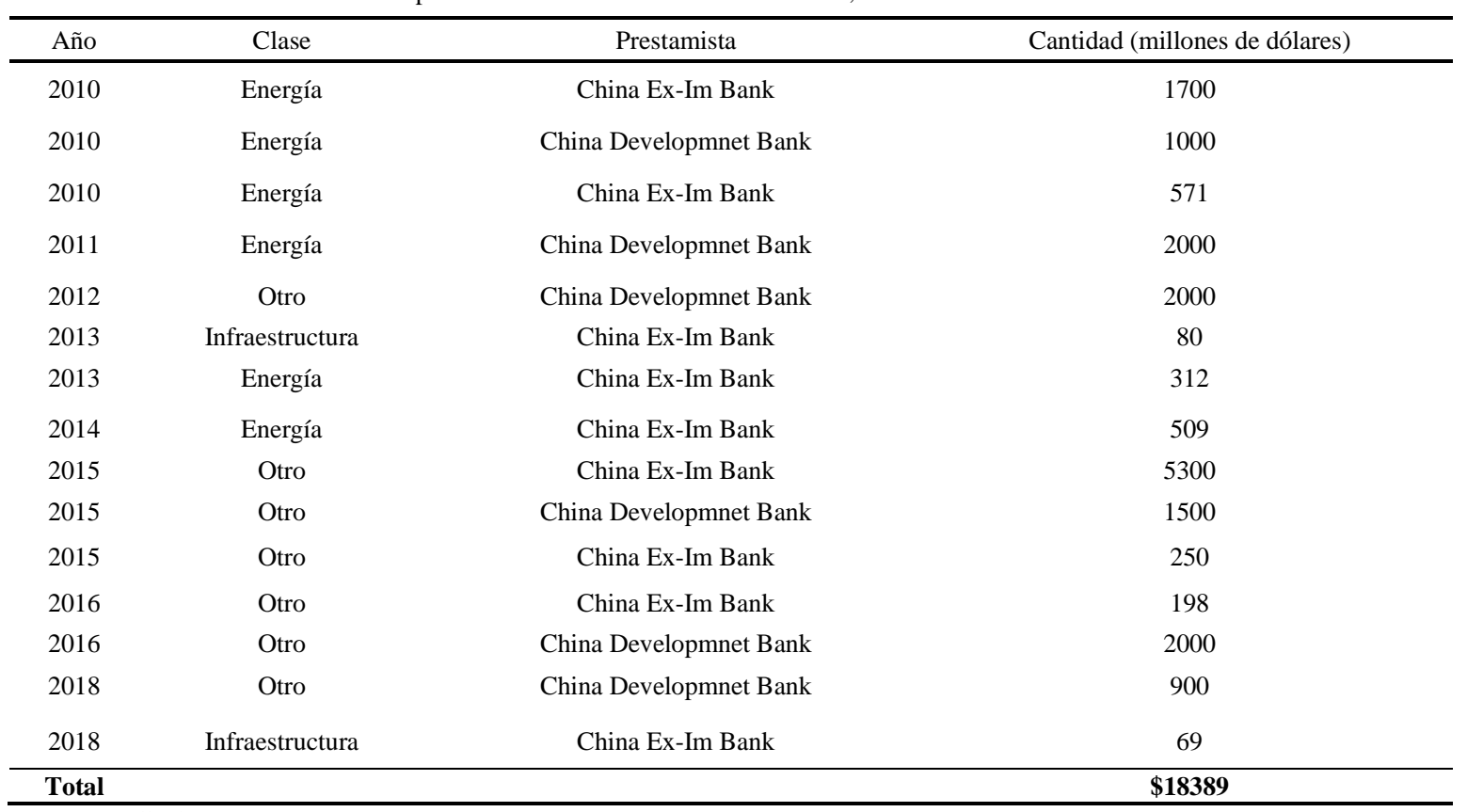

Source: Gallagher y Myers, 2020 